\title{
Squamosamide Derivative FLZ Protects Pancreatic $\beta$-Cells from Glucotoxicity by Stimulating Akt-FOXO1 Pathway
}

\author{
Xiangchen Kong, ${ }^{1}$ Longmei Zhang, ${ }^{1}$ Xianxin Hua, ${ }^{1,2}$ and Xiaosong $\mathrm{Ma}^{1}$ \\ ${ }^{1}$ Diabetes Center, Shenzhen University, Shenzhen 518060, China \\ ${ }^{2}$ University of Pennsylvania Perelman School of Medicine, Philadelphia, PA 19104, USA \\ Correspondence should be addressed to Xiaosong Ma; xsma@szu.edu.cn
}

Received 11 March 2015; Accepted 29 April 2015

Academic Editor: Shao-Nian Yang

Copyright (c) 2015 Xiangchen Kong et al. This is an open access article distributed under the Creative Commons Attribution License, which permits unrestricted use, distribution, and reproduction in any medium, provided the original work is properly cited.

\begin{abstract}
Chronic hyperglycemia increases apoptosis and reduces glucose-stimulated insulin secretion. Although protective agents have been searched extensively, none has been found so far. Here we tested FLZ, a synthetic derivative of squamosamide from a Chinese herb, as a potential candidate for antiglucotoxicity in INS-1E cells and mouse islets. Chronic culture of $\beta$-cells in $30 \mathrm{mM}$ glucose caused progressive reduction of cell viability, accompanied with increased apoptosis and reduced insulin secretion. These effects on apoptosis and insulin were reversed by FLZ in a dose-dependent manner. FLZ treatment also increased forkhead box O1 protein phosphorylation and reduced its nuclear location. On the contrary, FLZ increased pancreatic and duodenal homeobox-1 expression and its nuclear localization, an effect mediated by increased p-Akt. Consistently, Akt selective inhibitor MK-2206 completely abolished antiglucotoxicity effect of FLZ. Furthermore, FLZ treatment increased cytosolic ATP/ADP ratio. Taken together, our results suggest that FLZ could be a potential therapeutic agent to treat the hyperglycemia-induced $\beta$-cell failure.
\end{abstract}

\section{Introduction}

Chronic exposure of pancreatic $\beta$-cells to high glucose causes pancreatic $\beta$-cell failure characterized with an increase in apoptosis and a marked deficit of glucose-stimulated insulin secretion (GSIS) [1], termed glucotoxicity. Based on the failure of $\beta$-cell on the pathogenesis of type 2 diabetes, it is important to prevent and/or reverse hyperglycemia-impaired $\beta$-cell functions.

FLZ is a synthetic derivative (molecular weight of 458.2) of squamosamide and is formulated as N-[2-(4-hydroxyphenyl)-ethyl]-2-(2,5-dimethoxy-phenyl)-3-(3-methoxy-4hydroxy-p-henyl)-acrylamide. It has been found that FLZ has prominent antiapoptotic effect on dopaminergic neurons that are exposed to $\mathrm{MPP}^{+}[2,3]$ and 6-hydroxydopamine (6-OHDA) [4, 5] and also exerts neuroprotective effects on Parkinson's disease models [6]. The application for clinical trials of FLZ is under review by China Food and Drug Administration. Exactly how FLZ exerts its antiapoptotic effects remains unestablished but the effect may involve reducing mitochondrial oxidative stress [6]. Given that mitochondrial oxidative damage plays a key role in pancreatic $\beta$-cell failure in the pathogenesis of type 2 diabetes and given that mitochondria-targeted antioxidants protect pancreatic $\beta$-cells against oxidative stress under glucotoxic conditions [7], we therefore explored whether FLZ would prevent pancreatic $\beta$-cell glucotoxicity in this study.

\section{Materials and Methods}

2.1. Reagents and Antibodies. FLZ, a white powder with $99 \%$ purity, was kindly provided by Professor Dan Zhang from Institute of Materia Medica, Chinese Academy of Medical Sciences, and Peking Union Medical College. 3-(4,5-Dimethylthiazol-2-yl)-2,5-diphenyltetrazolium bromide (MTT), Hoechst 33342 (HO), propidium iodide (PI), sodium pyruvate, glutamine, and beta-mercaptoethanol were purchased from Sigma (St. Louis, MO, USA). MK-2206 was purchased from Selleck Chemicals (Houston, TX, USA). Collagenase P was provided by Roche Molecular Biochemicals (Indianapolis, IN, USA). PDX-1, Akt, Lamin B, and $\beta$-actin antibodies 
were purchased from Santa Cruz Biotechnology (CA, USA). p-Akt (Ser473), p-FOXO1 (Ser256), and FOXO1 antibodies were obtained from Cell Signaling (Danvers, MA, USA). Goat and rabbit secondary antibodies were product of Sigma (St. Louis, MO, USA). Rat and mouse Insulin Ultrasensitive ELISA kit were purchased from ALPCO Diagnostics (Salem, NH, USA). Nuclear-cytosol extraction kit was purchased from Applygen Technologies (Beijing, China). ADP/ATP assay kit was provided by BioVision (Mountain View, CA, USA).

2.2. Cell Culture. Rat pancreatic INS-1E cells were kindly provided by Professor Yong Liu from Shanghai Institute for Nutritional Sciences of Chinese Academy of Sciences. INS$1 \mathrm{E}$ cells between passages 60-90 were cultured in RPMI1640 medium, as reported previously [8].

2.3. Islet Isolation. Female C57BL/6 mice $(4 \mathrm{w})$ were purchased from the experimental animal centre of Guangdong Academy of Medical Science (Guangzhou, China). The mice were sacrificed by cervical dislocation. Pancreatic islets were isolated and cultured using the methods reported previously [8]. Use and care of mice for the experiments were approved by the ethical committee overseeing research involving animals at Shenzhen University.

2.4. Cell Viability. Cell viability was assessed with MTT assay. The assay is based on the ability of living cells to convert MTT into insoluble formazan. Thus, the amount of produced formazan is proportional to the number of living cells. In detail, INS-1E cells were seeded in 96-well plates and cultured in $11.1 \mathrm{mM}$ glucose (G11.1) or $30 \mathrm{mM}$ glucose (G30) in the presence or absence of different concentration of FLZ and with or without $50 \mathrm{nM} \mathrm{MK}-2206$ for $96 \mathrm{~h}$. Thereafter, $100 \mu \mathrm{L}$ of $0.5 \mathrm{mg} / \mathrm{mL}$ MTT was added to each well and incubated at $37^{\circ} \mathrm{C}$ for $4 \mathrm{~h}$. The resulting formazan was dissolved in $100 \mu \mathrm{L}$ DMSO. Samples were read at $570 \mathrm{~nm}$ using a microplate reader (BioTek Epoch, USA).

2.5. Apoptosis Assay. Apoptotic assay was carried out using Hoechst 33342 (HO) and propidium iodide (PI) staining method and counting cell numbers under a fluorescent microscope. In brief, after treating cells with $30 \mathrm{mM}$ glucose for $96 \mathrm{~h}$, the cells were incubated with $20 \mu \mathrm{g} / \mathrm{mL} \mathrm{HO}$ and $10 \mu \mathrm{g} / \mathrm{mL} \mathrm{PI}$ at $37^{\circ} \mathrm{C}, 5 \% \mathrm{CO}_{2}$ for $15 \mathrm{~min}$. Then the cells were washed once with PBS and the number of apoptotic cells was counted under an inverted fluorescent microscope. A minimum of 500 cells was counted for each plate under randomized conditions.

2.6. GSIS and Insulin Content Assay. INS-1E cells or mouse islets were seeded in 24-well plates and were cultured in $5.5 \mathrm{mM}$ glucose (G5.5) or $30 \mathrm{mM}$ glucose (G30) in the absence or presence of $10 \mu \mathrm{M}$ FLZ and with or without $50 \mathrm{nM}$ MK-2206 for $72 \mathrm{~h}$. Then insulin release from mouse islets and INS-1E cells was assayed as published [8]. In brief, the cells were preincubated in $1 \mathrm{~mL}$ Krebs Ringer bicarbonate (KRB) buffer for $1 \mathrm{~h}$ and then were stimulated with $1 \mathrm{~mL}$
KRB buffer containing $16.8 \mathrm{mM}$ glucose for $30 \mathrm{~min}$. The supernatants were collected for measurements of secreted insulin using Insulin Ultrasensitive ELISA kit. For INS-1E cells, the attached cells were harvested for determination of total cellular protein content. Insulin levels $(\mathrm{ng} / \mathrm{mL})$ were normalized against total cellular protein content in mg. For mouse islets, insulin levels $(\mathrm{ng} / \mathrm{mL})$ were normalized against islet number. For insulin content assay, after treatment with FLZ in the presence or absence of MK-2206 for $72 \mathrm{~h}$, the cells were lysed with RIPA lysis buffer. The insulin levels in the cell lysates were determined with Insulin Ultrasensitive ELISA kit. Insulin levels $(\mathrm{ng} / \mathrm{mL})$ were normalized against total cellular protein content in $\mathrm{mg}$.

2.7. Western Blotting. For extraction of total protein, cell pellets were incubated in RIPA lysis buffer supplemented with $1 \mathrm{mM}$ protease inhibitor cocktail for 30 minutes on ice, followed by centrifugation at $14,000 \mathrm{~g}$ for 10 minutes at $4^{\circ} \mathrm{C}$. Cytosolic and nuclear protein were prepared using a nuclearcytosol extraction kit according to the manufacturer's protocol. Cell lysates were separated using SDS-PAGE gels and transferred onto PVDF membrane. The membranes were immunoblotted with respective primary antibodies overnight at $4^{\circ} \mathrm{C}$ and then incubated with horseradish peroxidaseconjugated secondary antibody for $2 \mathrm{~h}$ at room temperature. The immunoreactive bands were visualized by the KODAK Image Station 4000MM PRO imaging system and the densities of the bands were determined using Gel-Pro Analyzer 4.0 software and normalized against the level of $\beta$-actin or Lamin B.

2.8. ATP/ADP Assay. INS-1E cells were seeded in 96-well plates and cultured in $5.5 \mathrm{mM}$ or $30 \mathrm{mM}$ glucose medium in the presence or absence of $10 \mu \mathrm{M}$ FLZ for $72 \mathrm{~h}$. After preincubation in $1 \mathrm{~mL} \mathrm{KRB}$ buffer for 1 hour at $37^{\circ} \mathrm{C}$, the cells were stimulated with $\mathrm{KRB}$ buffer containing $16.8 \mathrm{mM}$ glucose for $30 \mathrm{~min}$. Then ATP and ADP were measured using BioVision ADP/ATP Ratio Assay Kit according to the manufacturer's protocol.

2.9. Statistical Analyses. Data are presented as mean \pm S.E.M. for the indicated number of experiments $(n)$. Statistical significance was evaluated using the independent $t$-test or one-way ANOVA. Data were considered significant when $p<$ 0.05 .

\section{Results}

3.1. FLZ Protects INS-1E Cells and Mouse Islets from Glucotoxicity. Insulin-secreting INS-1E cells and mouse islets were cultured in $30 \mathrm{mM}$ glucose (G30) for 3 to 5 days, a commonly used model for glucotoxicity $[9,10]$. As shown in Figure 1(a), chronic culture of INS-1E cells in G30 resulted in marked decrease in cell viability as assayed by MTT and the value reduced by $\sim 25 \%$ (3 days), $\sim 52 \%$ ( 4 days), and $\sim 75 \%$ (5 days) compared to that cultured in $11.1 \mathrm{mM}$ glucose (G11.1), as reported previously [11]. Treatment with increasing concentrations of $\operatorname{FLZ}(0.1,1,10$, and $20 \mu \mathrm{M})$ 

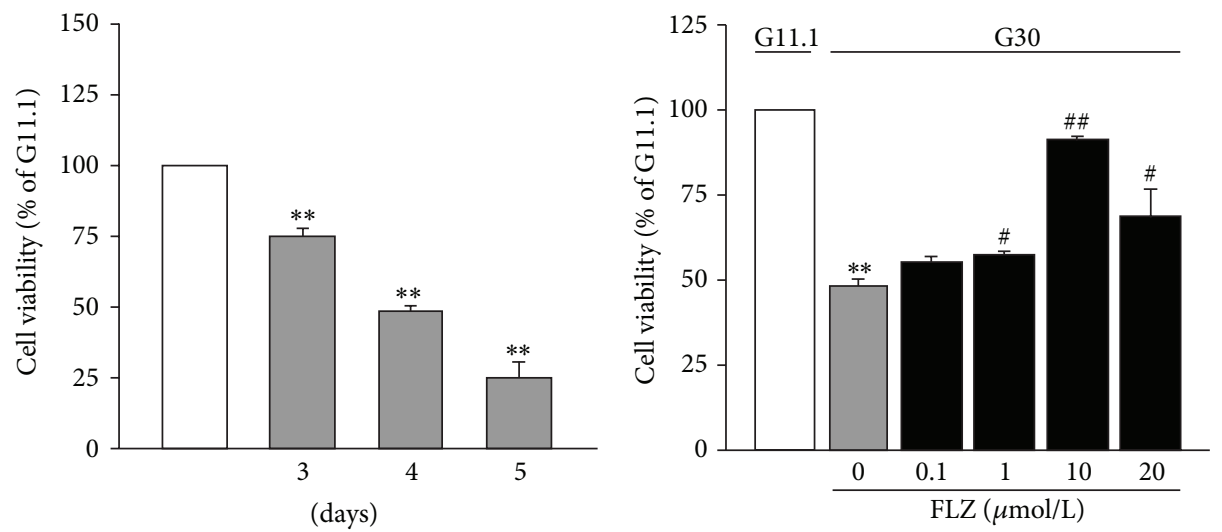

(a)

(b)
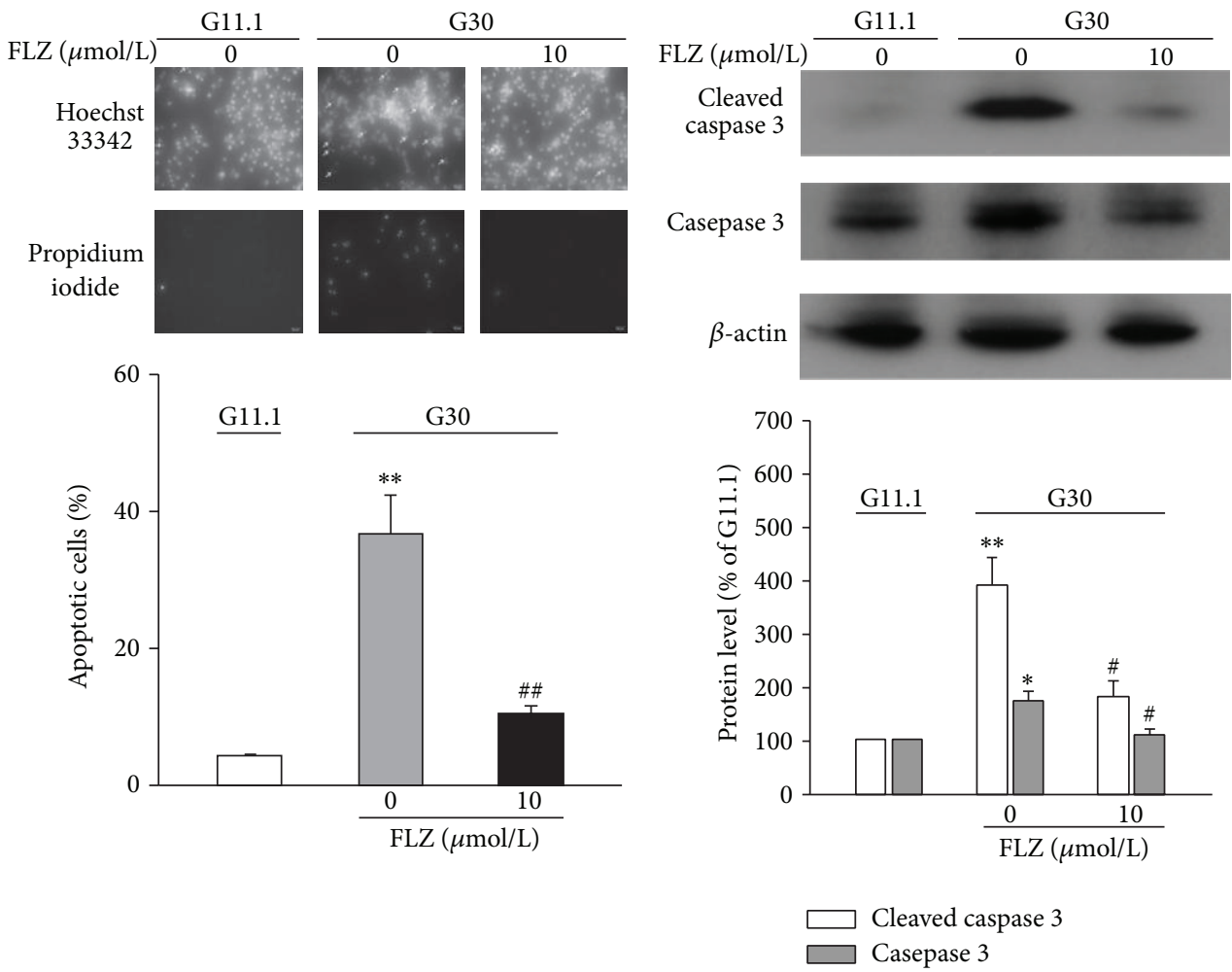

(c)

(d)

Figure 1: Treatment with FLZ prevents glucotoxicity in INS-1E cells. (a) INS-1E cells were cultured at G11.1 and G30 for 3-5 days and cell viability was assessed with MTT assay. Values were expressed as percentage of cell viability at G11.1. Data are means \pm S.E.M. of 3-6 independent experiments. ${ }^{* *} p<0.01$ versus the value at G11.1. (b) Cell viability was assessed in INS-1E cells being cultured at G11.1 and G30 for 4 days. Cells were treated without or with varying levels of FLZ. Values were expressed as percentage of cell viability at G11.1. Data are means \pm S.E.M. of 3 independent experiments. ${ }^{* *} p<0.01$ versus the value at G11.1; ${ }^{\#} p<0.05,{ }^{\# \#} p<0.01$ versus the value at G30 without FLZ. (c) Apoptosis assessed by HO/PI staining in INS-1E cells cultured at G11.1 and G30 for 4 days. Cells were treated without or with $10 \mu \mathrm{M}$ FLZ. Arrows indicate HO-stained cells with apoptotic bodies (upper panel). Mean values \pm S.E.M. of 3 independent experiments (low panel). ${ }^{* *} p<0.01$ versus the value at G11.1; ${ }^{\# \#} p<0.01$ versus the value at G30 without FLZ. (d) INS-1E cells were cultured at G11.1 or G30 for 4 days. Cells were treated without or with $10 \mu \mathrm{M}$ FLZ. Expression of total and cleaved caspase 3 protein was determined by western blot. $\beta$-actin was used as an internal control (upper panel). Intensities of total and cleaved caspase 3 protein expression were quantified, normalized against the level of $\beta$-actin, and expressed as fold of protein abundance in INS-1E cells at G11.1 (low panel). Data are means \pm S.E.M. of 3 separate experiments. ${ }^{*} p<0.05,{ }^{* *} p<0.01$ versus the value at G11.1; ${ }^{\#} p<0.05$ versus the value at G30 without FLZ. 
reversed the effect of $30 \mathrm{mM}$ glucose in a dose-dependent manner (Figure 1(b)). A concentration of $10 \mu \mathrm{M}$ of FLZ was used in most of experiments of this study, as this concentration corresponds to a maximal effective concentration reported in other studies [6]. The effect of FLZ on $\beta$-cell glucotoxicity was also investigated by measuring apoptotic cells as determined by HO/PI staining (Figure 1(c)). Chronic culture at G30 led to an increase in the percentages of apoptotic cells from $4 \pm 0.1 \%$ to $37 \pm 6 \%$ at G11.1, whereas FLZ treatment reduced the level of apoptosis to $10 \pm 1 \%$ $(p<0.01)$ (Figure 1(c)). Notably, addition of FLZ essentially blocked glucotoxicity-induced increase of cleaved caspase 3 (Figure 1(d)), an activated proapoptotic protein. Thus, FLZ protects $\beta$-cells from glucotoxicity-induced cell death at least partly by preventing activation of caspase 3 .

\subsection{FLZ Increases Insulin Content and Secretion at Glucotoxic-} ity. To determine whether treatment with FLZ has beneficial effects on insulin content and GSIS, INS-1E cells and mouse islets were cultured at G5.5 and G30 for 3 days. As shown in Figures 2(a)-2(c), insulin content (Figure 2(a)) and GSIS (Figures 2(b) and 2(c)) were decreased to $\sim 25 \%, \sim 45 \%$, and $\sim 25 \%$, respectively, after being cultured at G30 for 3 days, as compared to those cultured in low glucose (G5.5). In contrast, treatment with $10 \mu \mathrm{M}$ FLZ partially but significantly reversed glucotoxicity-induced reduction of insulin content dosedependently (Figure 2(a)), as well as glucotoxicity-induced decrease of GSIS $(p<0.01)$ in INS-1E cells (Figure 2(b)) and mouse islets (Figure 2(c)).

3.3. FLZ Restored Glucotoxicity-Altered Expression and Nuclear Localization of PDX-1 and FOXO1. It has been reported that glucotoxicity suppresses the expression and nuclear localization of the pancreatic and duodenal homeobox-1 (PDX-1) [12]. Consistently, our data show that chronic exposure of $\beta$-cells to G30 reduced PDX-1 expression (Figure 3(a)) and its nuclear localization (Figure 3(b)), accompanied by increased cytosolic distribution (see Suppl. Figure 1 in Supplementary Material available online at http://dx.doi.org/10.1155/2015/803986). In contrast, culture at $\mathrm{G} 30$ for $72 \mathrm{~h}$ resulted in reduction of phosphorylated forkhead box O1 protein (FOXO1) (Figure 3(c)) and its cytosolic distribution (Suppl. Figure 2), associated with increased nuclear distribution (Figure 3(d)). Notably, treatment with FLZ increased the PDX-1 expression and its nuclear localization (Figures 3(a) and 3(b)), accompanied by reduced cytosolic localization (Suppl. Figure 1), whereas treatment with FLZ increased phosphorylated level of FOXO1 (Figure 3(c)) and reduced its nuclear presence (Figure 3(d)), associated with its increased cytosolic localization (Suppl. Figure 2).

3.4. The Antiglucotoxic Effect of FLZ Is Mediated via Akt. Akt is an important prosurvival kinase. We next explored whether the antiglucotoxic effect of FLZ is involved in regulating activation of Akt. The results showed that FLZ increased the phosphorylation of Akt (p-Akt) in a dose-dependent manner (Figure 4(a)). As reported in another study [13], incubation of cells at G30 for 4 days essentially reduced the level of phosphorylated Akt, as compared to that at G11.1. It is of note that treatment with $10 \mu \mathrm{M}$ FLZ significantly increased the level of p-Akt $(p<0.01)$ (Figure 4(b)). In the presence of the Akt inhibitor MK-2206 (50 nM), which has no effect on G30 condition (Suppl. Figure 4), FLZ lost its ability to reduce the effects of glucotoxicity on cell viability (Figure 4(c)), insulin content (Figure 4(d)), and GSIS (Figure 4(e)). In addition, the Akt-mediated effect of FLZ was further confirmed by the data showing that the effect of FLZ on PDX-1 was completely abolished by MK-2206 (Suppl. Figures 3A-3C).

Based on the observations that FLZ protected dopaminergic neurons against apoptosis via reducing mitochondrial oxidative stress [6], we next explored whether the fact that FLZ prevents glucotoxicity-induced $\beta$-cell failure would be due to improvement of mitochondrial function. INS-1E cells were cultured at G11.1 and G30 mM for 4 days and treated without or with $10 \mu \mathrm{M}$ FLZ. As shown in Figure 5, chronic culture at G30 substantially reduced the level of ATP/ADP ratio, consistent with previous reports [8]. Treatment with FLZ significantly increased ATP/ADP ratio.

\section{Discussion}

In the present study, we provide evidence that the synthetic derivative of squamosamide FLZ prevents glucotoxicityinduced $\beta$-cell dysfunction by a mechanism involving Akt and its downstream target FOXO1.

FLZ is a synthetic derivative of squamosamide from a Chinese herb and has been found to be capable of protecting dopaminergic cells against $\mathrm{MPP}^{+}-[2,3]$ and 6-OHDA- [4, 5] induced apoptosis, via activation of PI3K/Akt signaling pathway $[2,6]$. Like its effects found in dopaminergic neurons, FLZ protects $\beta$-cells against glucotoxicity-induced apoptosis through preventing activation of caspase 3 induced by glucotoxicity (Figure $1(\mathrm{~d})$ ) and thus increases cell viability in a dose-dependent manner (Figures $1(\mathrm{a})-1(\mathrm{c})$ ). In addition, we also show that treatment with FLZ significantly decreases glucotoxicity-induced reduction of insulin content (Figure 2(a)), as well as glucose-stimulated insulin secretion in INS-1E cells and mouse islets (Figures 2(b) and 2(c)). Given that reduced $\beta$-cell mass and GSIS by chronic hyperglycemia are essential for development of T2D [14], this antiglucotoxic effect of FLZ is of vital merit for treatment of T2D.

The pancreatic and duodenal homeobox factor-1 (PDX1) plays a crucial role in maintaining mature $\beta$-cell function, such as transactivating the insulin gene and the genes controlling GLUT2 and glucokinase that are important for glucose sensing and metabolism $[15,16]$. Indeed, heterozygous mutation of PDX-1 causes glucose intolerance, associated with increased islet apoptosis and impaired GSIS, which collectively results in maturity onset diabetes $[16,17]$. In pancreatic $\beta$-cells, PDX-1 is negatively regulated by FOXO1 [18]; the latter acts as a transcriptional brake on PDX-1 [19]. Moreover, FOXO1 and PDX-1 are mutually exclusive patterns of nuclear location in $\beta$-cells [19]. Thus, FOXO1 inactivation leads to FOXO1 nuclear exclusion and PDX-1 expression and finally $\beta$-cell proliferation and insulin gene 


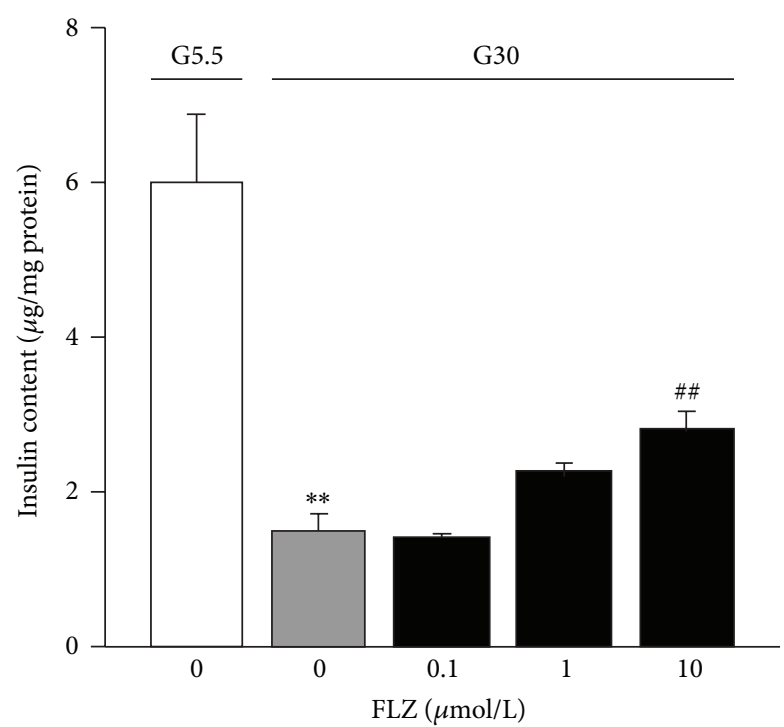

(a)

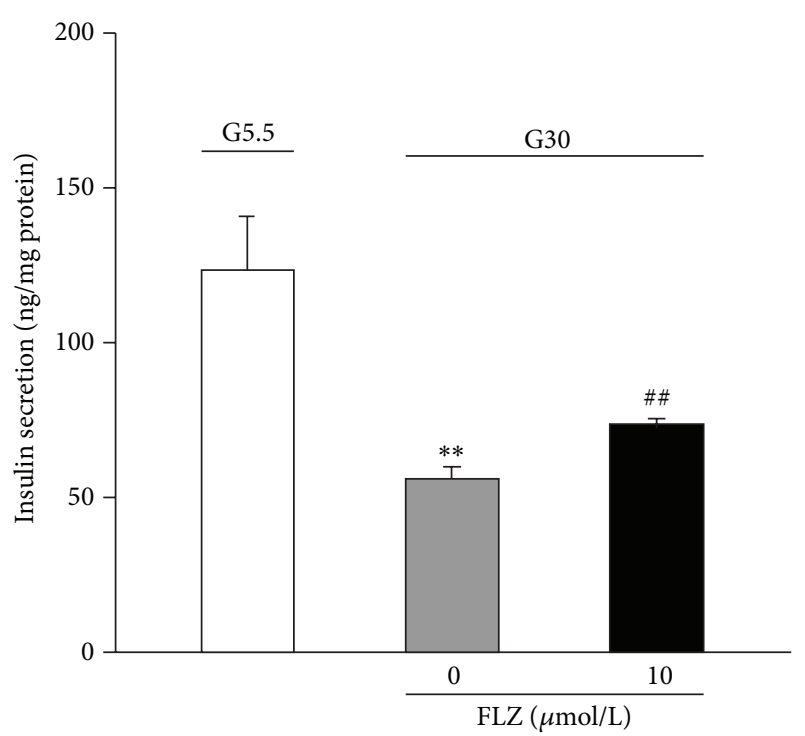

(b)

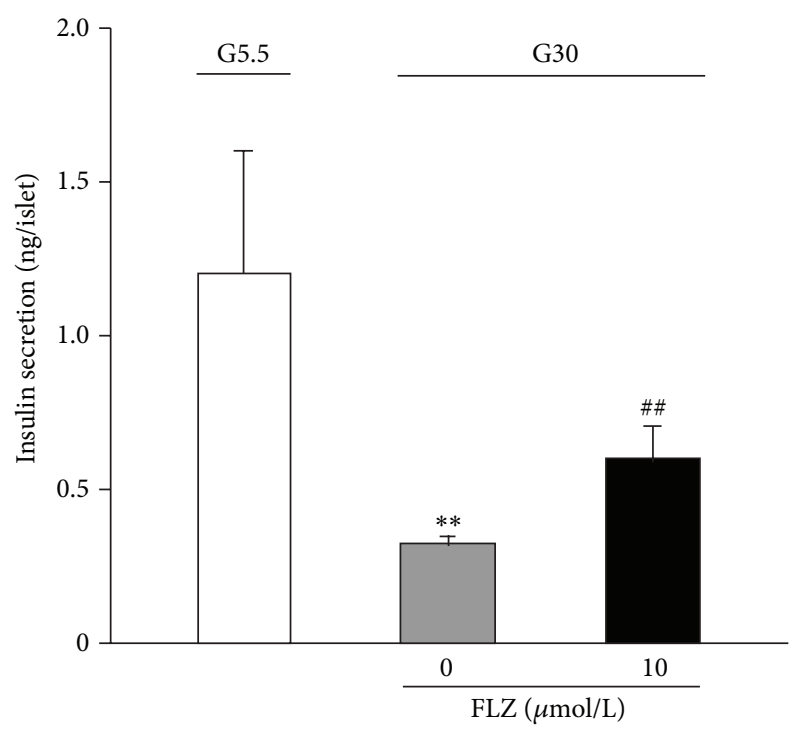

(c)

FIGURE 2: Treatment with FLZ increases insulin content and GSIS at glucotoxicity. (a) INS-1E cells were cultured at G5.5 or G30 for 3 days. Insulin content was assayed without or with varying level of FLZ. Values are normalized against total protein. Data are means \pm S.E.M. of 5-8 experiments for each group. ${ }^{* *} p<0.01$ versus the value at G5.5; ${ }^{\# \#} p<0.01$ versus the value at G30 without FLZ. ((b), (c)) Insulin secretion in INS-1E cells (b) or mouse islets (c) cultured at G5.5 (white bar) or G30 (grey bar) with or without $10 \mu \mathrm{M}$ FLZ (black bar) for 3 days. After preincubation with $1 \mathrm{~mL} \mathrm{KRB} \mathrm{buffer} \mathrm{for} 1 \mathrm{~h}$, the cells were stimulated with $1 \mathrm{~mL} \mathrm{KRB}$ buffer containing $16.8 \mathrm{mM}$ glucose for $30 \mathrm{~min}$. The supernatants were collected for measurement of secretory insulin. Values were normalized against total protein (b) or the amount of insulin secretion per islet (c); data are means \pm S.E.M. of 7-11 (b) or of 5-8 experiments (c). ${ }^{* *} p<0.01$ versus the value at G5.5; ${ }^{\# \#} p<0.01$ versus the value at G30 without FLZ.

expression $[19,20]$. Our data extend the previous observations by showing that chronic exposure of $\beta$-cells to elevated glucose stimulates FOXO1 activation and increases its nuclear location (Figures 3(c) and 3(d)) and therefore results in reduced expression and nuclear location of PDX-1 (Figures 3(a) and 3(b)) and finally reduction of insulin content, $\beta$ cell mass, and insulin secretion. However, treatment with FLZ reverses the detrimental effect of glucotoxicity on PDX1 . Therefore, these data suggest that FLZ improves $\beta$-cell mass and function via regulation of FOXO1 and PDX-1 pathway.
In addition, it is known that pancreatic $\beta$-cells convert glucose to ATP and thereby lead to increase of the cellular ATP/ADP ratio and promote the closure of the ATP-sensitive, potassium channels ( $\mathrm{K}_{\text {ATP }}^{+}$channels) which is the initial triggering event for insulin secretion [21]. Therefore, the effect of FLZ on ATP/ADP ratio (Figure 5) may also be involved in its improvement in insulin secretion in $\beta$-cells at glucotoxicity.

It is known that FOXO1 is inhibited by Akt upon phosphorylation [19]. Activation of Akt-FOXO1 results in nuclear 

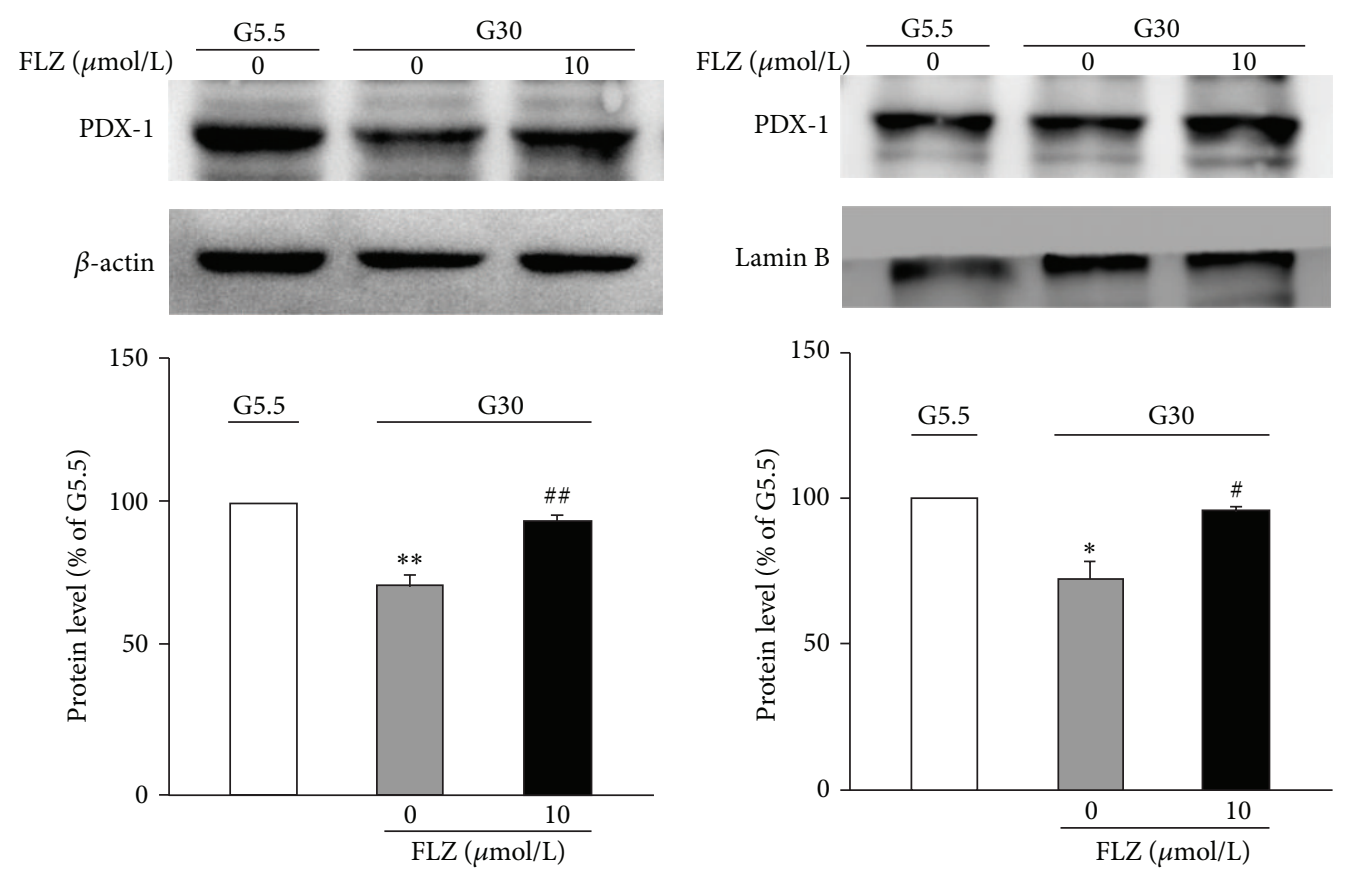

(a)
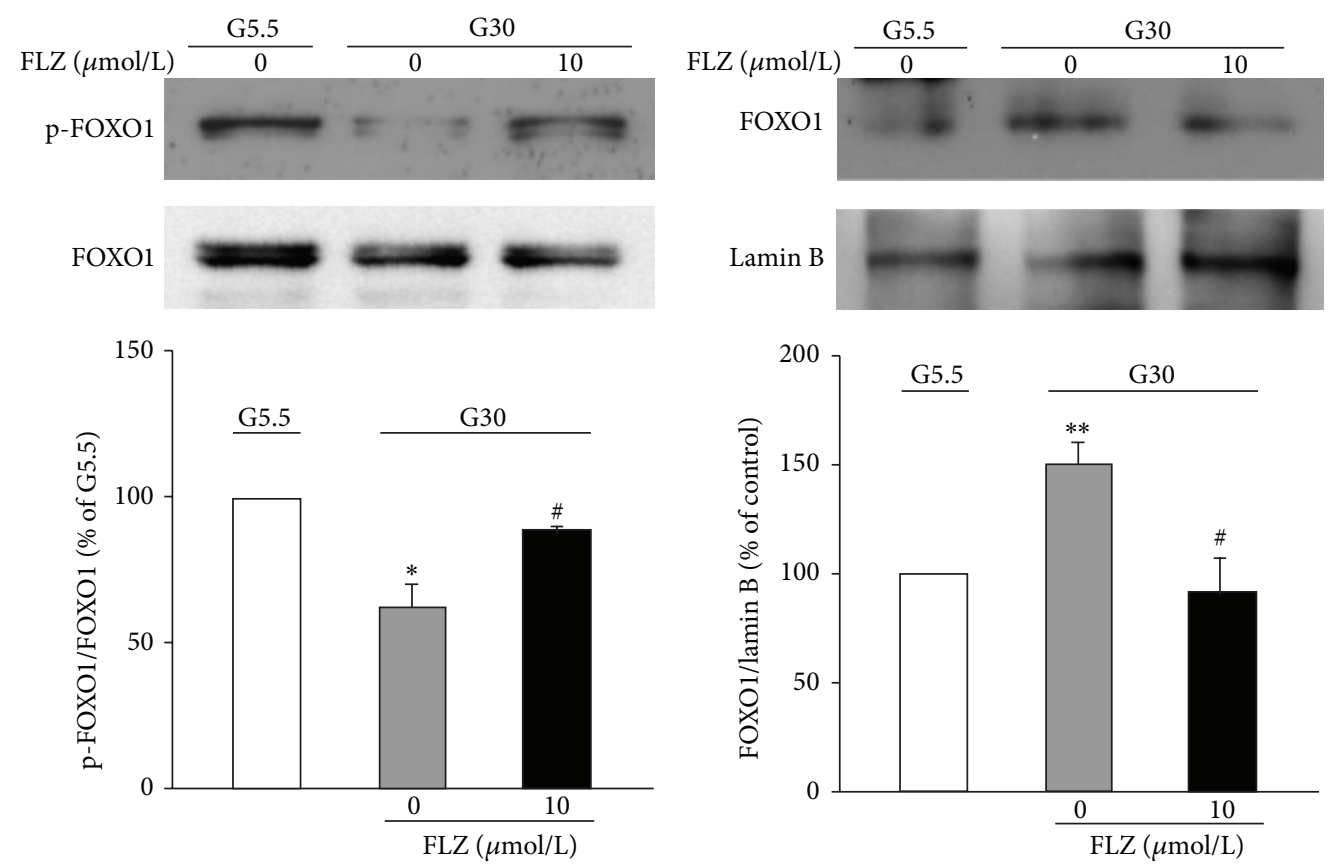

(c)
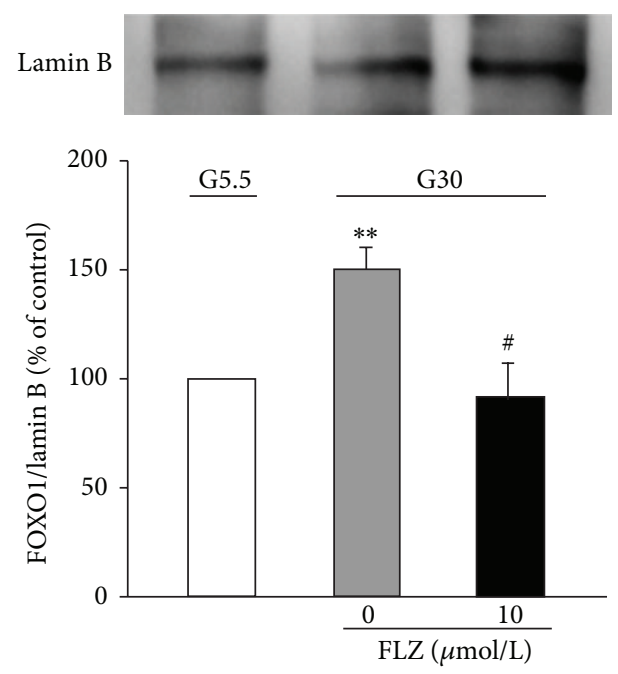

(d)

FIGURE 3: FLZ restores glucotoxicity-altered expression and intracellular localization of PDX-1 and FOXO1. ((a), (c)) INS-1E cells were cultured at G5.5 or G30 for 3 days. Cells were treated without or with $10 \mu \mathrm{M}$ FLZ. Expression of PDX-1 (a) or pFOXO1 (c) protein was determined by western blot. $\beta$-actin was used as an internal control ((a), upper panel). Intensities of PDX-1 (a) or pFOXO1 (c) protein expression were quantified, normalized against the level of $\beta$-actin (a) or total FOXO1 (c), and expressed as fold of protein abundance in INS-1E cells at G5.5 (low panels). Data are means \pm S.E.M. of 3 separate experiments each. ${ }^{* *} p<0.01$ versus the value at G5.5; ${ }^{\# \#} p<0.05$ versus the value at G30 without FLZ. ((b), (d)) INS-1E cells were cultured at G5.5 or G30 for 3 days. Cells were treated without or with $10 \mu \mathrm{M}$ FLZ. Nuclear protein extraction was subjected to western blot analysis. Lamin B was used as an internal control (upper panels). Intensities of PDX-1 (b) or FOXO1 (d) protein expression were quantified, normalized against the level of Lamin B, and expressed as fold of protein abundance in INS-1E cells at G5.5 (low panels). Means \pm S.E.M. results of 3 separate experiments each. ${ }^{*} p<0.05,{ }^{* *} p<0.01$ versus the value at G5.5; ${ }^{\#} p<0.05$ versus the value at G30 without FLZ. 


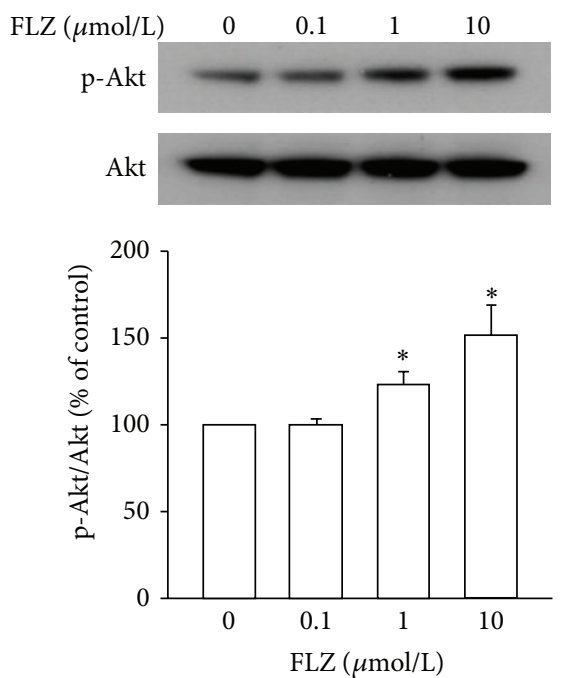

(a)

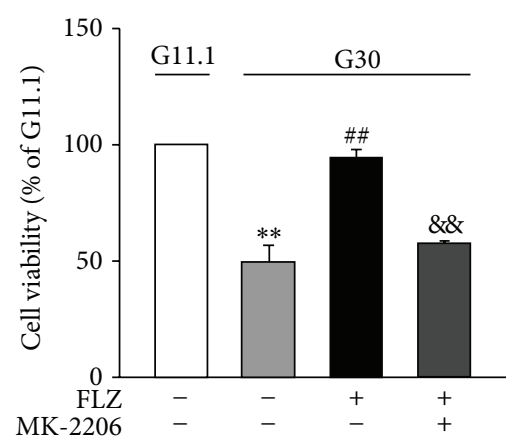

(c)
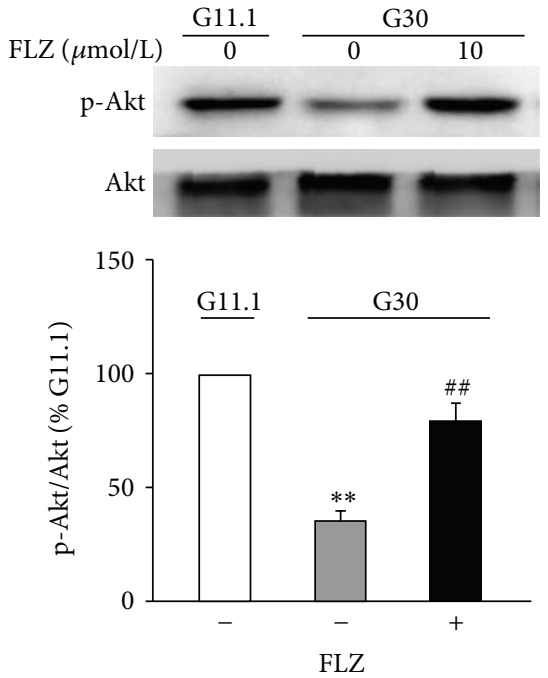

(b)

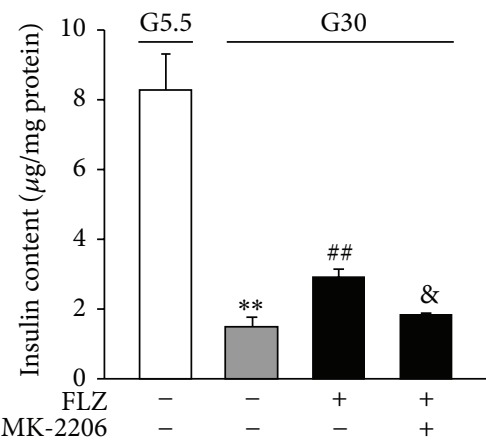

(d)

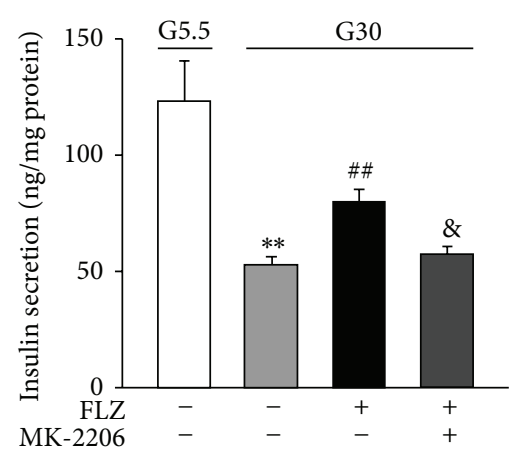

(e)

FIGURE 4: FLZ acts via stimulation of Akt. (a) INS-1E cells were treated with $0.1 \mu \mathrm{M}, 1 \mu \mathrm{M}$, and $10 \mu \mathrm{M}$ FLZ for $8 \mathrm{~h}$. Expression of p-Akt and total Akt protein was determined by western blot. Mean \pm S.E.M. of three separate experiments. Intensities of p-Akt protein expression were quantified, normalized against the level of total Akt, and expressed as fold of protein abundance in INS-1E cells treated with DMSO. ${ }^{*} p<0.05$ versus control. (b) INS-1E cells were cultured at G11.1 or G30 for 4 days. Cells were treated without or with $10 \mu \mathrm{M}$ FLZ. Expression of p-Akt and total Akt protein was determined by western blot. Mean \pm S.E.M. of seven separate experiments. ${ }^{* *} p<0.01$ versus the value at G11.1; $\# p<0.01$ versus the value at G30 without FLZ. (c) Cell viability was assessed in INS-1E cells being cultured at G11.1 and G30 for 4 days. Cells were treated without or with $10 \mu \mathrm{M}$ FLZ or simultaneous addition of $10 \mu \mathrm{M}$ FLZ and $50 \mathrm{nM}$ MK-2206. Values were expressed as percentage of cell viability at G11.1. Data are means \pm S.E.M. of 3-4 independent experiments. ${ }^{* *} p<0.01$ versus the value at G5.5; ${ }^{\# \#} p<0.01$ versus the value at G30 without FLZ; ${ }^{\& \&} p<0.01$ versus the value at G30 with FLZ. (d) Insulin content was assayed without or with $10 \mu \mathrm{M}$ FLZ or simultaneous addition of $10 \mu \mathrm{M}$ FLZ and $50 \mathrm{nM}$ MK-2206. Values are normalized against total protein. Data are means \pm S.E.M. of 4-12 experiments. ${ }^{* *} p<0.01$ versus the value at G5.5; ${ }^{\# \#} p<0.01$ versus the value at G30 without FLZ; ${ }^{*} p<0.05$ versus the value at G30 with FLZ. (e) Insulin secretion was assayed in INS-1E cells treated without or with $10 \mu \mathrm{M}$ FLZ or simultaneous addition of $10 \mu \mathrm{M}$ FLZ and $50 \mathrm{nM}$ MK-2206. Values were normalized against total protein. Data are mean \pm S.E.M. of 5-16 separate experiments. ${ }^{* *} p<0.01$ versus the value at G5.5; ${ }^{\# \#} p<0.01$ versus the value at G30 without FLZ; ${ }^{\circledR} p<0.05$ versus the value at G30 with FLZ. 


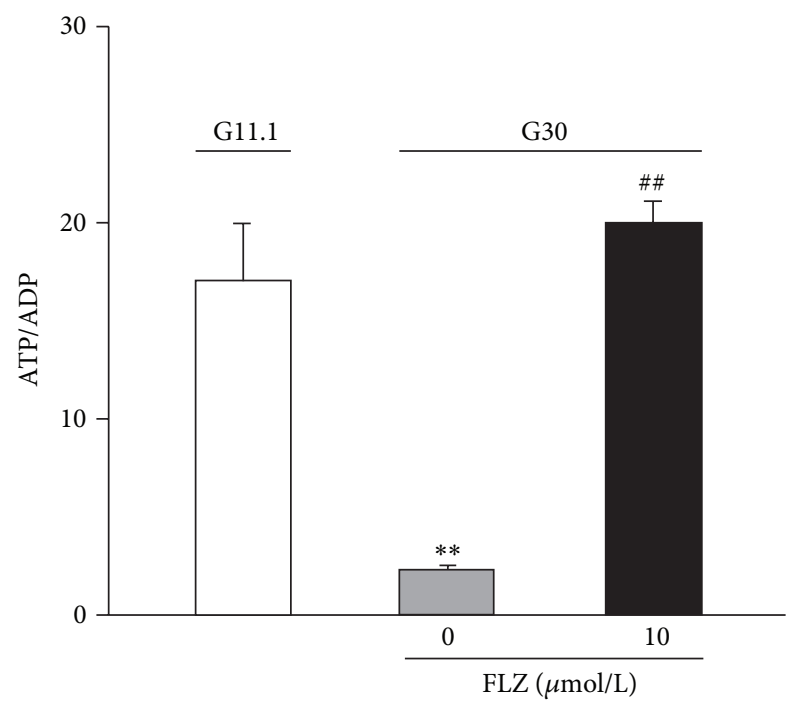

Figure 5: FLZ increases cytosolic ATP/ADP ratio at glucotoxicity. INS-1E cells were cultured at G11.1 or G30 for 3 days. Cells were treated without or with $10 \mu \mathrm{M}$ FLZ. Data are mean \pm S.E.M. of seven independent experiments. ${ }^{* *} p<0.01$ versus the value at G5.5; $\#$ \# $p<0.01$ versus the value at G30 without FLZ.

exclusion of FOXO1 and subsequently induces transcription of PDX-1 [19]. Akt/PKB is an important prosurvival kinase and is essential for maintaining $\beta$-cell mass and function. Our data reveal for the first time that FLZ prevents glucotoxicityinduced $\beta$-cell failure, an effect via activation of Akt. Three pieces of evidence corroborate this notion. First, FLZ stimulated Akt activation (Figures 4(a) and 4(b)). Second, treatment of INS-1E cells with Akt inhibitor MK-2206 almost abolished FLZ increased cell viability (Figure 4(c)), insulin content (Figure 4(d)), and GSIS (Figure 4(e)). Third, while FLZ was capable of reversing glucotoxicity-altered expression and nuclear location of PDX-1 (Figures 3(a) and 3(b)), FLZ failed to do so in the presence of Akt inhibitor MK-2206 (Suppl. Figures 3A and 3B).

The detailed mechanisms as to how FLZ leads to phosphorylation of Akt require future investigation. However, our results imply that the FLZ-induced Akt activation may be attributed to its ability of restoring mitochondrial function impaired by glucotoxicity, given FLZ increased cytosolic ATP/ADP ratio diminished by glucotoxicity (Figure 5). This notion is in fact supported by the observation that blocking mitochondrial fission with subsequent reduction in $\mathrm{H}_{2} \mathrm{O}_{2}$ increased insulin-induced Akt/PKB phosphorylation [22].

In conclusion, our data demonstrate that FLZ prevents glucotoxicity-induced $\beta$-cell dysfunction. The mechanism involved could imply Akt activation and sequentially phosphorylates FOXO1 and leads to its nuclear exclusion and consequently upregulates transcription of PDX-1 [19]. This cascade of events will finally result in increased insulin production and $\beta$-cell mass. Taken together, our results suggest that FLZ could serve as a potential therapeutic agent for the treatment of hyperglycemia-induced $\beta$-cell failure.

\section{Conflict of Interests}

There is no conflict of interests associated with this paper.

\section{Authors' Contribution}

Xiaosong Ma and Xiangchen Kong designed research; Xiangchen Kong performed the research and analyzed data. Longmei Zhang performed research. Xianxin Hua contributed to discussion and interpretation of the data. Xiaosong Ma and Xiangchen Kong wrote the paper.

\section{Acknowledgments}

This work was supported by the National Natural Science Foundation of China (Grants nos. 81400801, Xiangchen Kong, and 81370914, Xiaosong Ma), Shenzhen Scientific and Innovative Committee (Grants nos. KQC201108300039A and JCYJ20120613161323514, Xiaosong Ma, and JCYJ20130326112700890, Xiangchen Kong), and China Postdoctoral Science Foundation (Grant no. 2013M531877, Xiangchen Kong).

\section{References}

[1] S. Marshak, G. Leibowitz, F. Bertuzzi et al., "Impaired $\beta$-cell functions induced by chronic exposure of cultured human pancreatic islets to high glucose," Diabetes, vol. 48, no. 6, pp. 1230-1236, 2000.

[2] D. Zhang, J.-J. Zhang, and G.-T. Liu, "The novel squamosamide derivative (compound FLZ) attenuated 1-methyl, 4-phenylpyridinium ion (MPP+)-induced apoptosis and alternations of related signal transduction in SH-SY5Y cells," Neuropharmacology, vol. 52, no. 2, pp. 423-429, 2007.

[3] X.-C. Kong, D. Zhang, C. Qian, G.-T. Liu, and X.-Q. Bao, "FLZ, a novel HSP27 and HSP70 inducer, protects SH-SY5Y cells from apoptosis caused by $\mathrm{MPP}^{+}$," Brain Research, vol. 1383, pp. 99107,2011

[4] D. Zhang, J.-J. Zhang, and G.-T. Liu, "The novel squamosamide derivative FLZ protects against 6-hydroxydopamine-induced apoptosis through inhibition of related signal transduction in SH-SY5Y cells," European Journal of Pharmacology, vol. 561, no. 1-3, pp. 1-6, 2007.

[5] X.-Q. Bao, X.-C. Kong, L.-B. Kong, L.-Y. Wu, H. Sun, and D. Zhang, "Squamosamide derivative FLZ protected dopaminergic neuron by activating Akt signaling pathway in 6-OHDAinduced in vivo and in vitro Parkinson's disease models," Brain Research, vol. 1547, pp. 49-57, 2014.

[6] X.-Q. Bao, X.-C. Kong, C. Qian, and D. Zhang, "FLZ protects dopaminergic neuron through activating protein kinase $\mathrm{B} /$ mammalian target of rapamycin pathway and inhibiting RTP801 expression in Parkinson's disease models," Neuroscience, vol. 202, pp. 396-404, 2012.

[7] S. Lim, M. A. Rashid, M. Jang et al., "Mitochondria-targeted antioxidants protect pancreatic $\beta$-cells against oxidative stress and improve insulin secretion in glucotoxicity and glucolipotoxicity," Cellular Physiology and Biochemistry, vol. 28, no. 5, pp. 873-886, 2011.

[8] G. Luo, X. Kong, L. Lu, X. Xu, H. Wang, and X. Ma, “Glucagonlike peptide 1 potentiates glucotoxicity-diminished insulin 
secretion via stimulation of cAMP-PKA signaling in INS-1E cells and mouse islets," International Journal of Biochemistry and Cell Biology, vol. 45, no. 2, pp. 483-490, 2013.

[9] B. Roger, J. Papin, P. Vacher et al., "Adenylyl cyclase 8 is central to glucagon-like peptide 1 signalling and effects of chronically elevated glucose in rat and human pancreatic beta cells," Diabetologia, vol. 54, no. 2, pp. 390-402, 2011.

[10] H. K. Nyblom, E. Sargsyan, and P. Bergsten, "AMP-activated protein kinase agonist dose dependently improves function and reduces apoptosis in glucotoxic $\beta$-cells without changing triglyceride levels," Journal of Molecular Endocrinology, vol. 41, no. 3, pp. 187-194, 2008.

[11] Z.-Q. Hou, H.-L. Li, L. Gao, L. Pan, J.-J. Zhao, and G.-W. Li, "Involvement of chronic stresses in rat islet and INS-1 cell glucotoxicity induced by intermittent high glucose," Molecular and Cellular Endocrinology, vol. 291, no. 1-2, pp. 71-78, 2008.

[12] K.-G. Park, K.-M. Lee, H.-Y. Seo et al., "Glucotoxicity in the INS-1 rat insulinoma cell line is mediated by the orphan nuclear receptor small heterodimer partner," Diabetes, vol. 56, no. 2, pp. 431-437, 2007.

[13] O. Kluth, F. Mirhashemi, S. Scherneck et al., "Dissociation of lipotoxicity and glucotoxicity in a mouse model of obesity associated diabetes: role of forkhead box O1 (FOXO1) in glucose-induced beta cell failure," Diabetologia, vol. 54, no. 3, pp. 605-616, 2011.

[14] Y. Zhang and D. Liu, "Flavonol kaempferol improves chronic hyperglycemia-impaired pancreatic beta-cell viability and insulin secretory function," European Journal of Pharmacology, vol. 670, no. 1, pp. 325-332, 2011.

[15] H. Watada, Y. Kajimoto, Y. Umayahara et al., "The human glucokinase gene beta-cell-type promoter: an essential role of insulin promoter factor 1/PDX-1 in its activation in HIT-T15 cells," Diabetes, vol. 45, no. 11, pp. 1478-1488, 1996.

[16] U. Ahlgren, J. Jonsson, L. Jonsson, K. Simu, and H. Edlund, “ $\beta$ cell-specific inactivation of the mouse $I p f 1 / P d x 1$ gene results in loss of the $\beta$-cell phenotype and maturity onset diabetes," Genes and Development, vol. 12, no. 12, pp. 1763-1768, 1998.

[17] M. Brissova, M. Shiota, W. E. Nicholson et al., "Reduction in pancreatic transcription factor PDX-1 impairs glucosestimulated insulin secretion," The Journal of Biological Chemistry, vol. 277, no. 13, pp. 11225-11232, 2002.

[18] Z. Meng, J. Lv, Y. Luo et al., "Forkhead box O1/pancreatic and duodenal homeobox 1 intracellular translocation is regulated by c-Jun $\mathrm{N}$-terminal kinase and involved in prostaglandin $\mathrm{E}_{2}$ induced pancreatic $\beta$-cell dysfunction," Endocrinology, vol. 150, no. 12, pp. 5284-5293, 2009.

[19] T. Kitamura, J. Nakae, Y. Kitamura et al., "The forkhead transcription factor Foxol links insulin signaling to Pdx1 regulation of pancreatic $\beta$ cell growth," The Journal of Clinical Investigation, vol. 110, no. 12, pp. 1839-1847, 2002.

[20] H. Kaneto, T.-A. Matsuoka, T. Miyatsuka et al., "PDX-1 functions as a master factor in the pancreas," Frontiers in Bioscience, vol. 13, no. 16, pp. 6406-6420, 2008.

[21] A. R. Meloni, M. B. DeYoung, C. Lowe, and D. G. Parkes, "GLP1 receptor activated insulin secretion from pancreatic beta-cells: mechanism and glucose dependence," Diabetes, Obesity and Metabolism, vol. 15, no. 1, pp. 15-27, 2013.

[22] M. E. Smith, T. S. Tippetts, E. S. Brassfield et al., "Mitochondrial fission mediates ceramide-induced metabolic disruption in skeletal muscle," Biochemical Journal, vol. 456, no. 3, pp. 427439, 2013. 


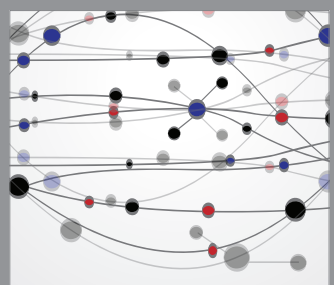

The Scientific World Journal
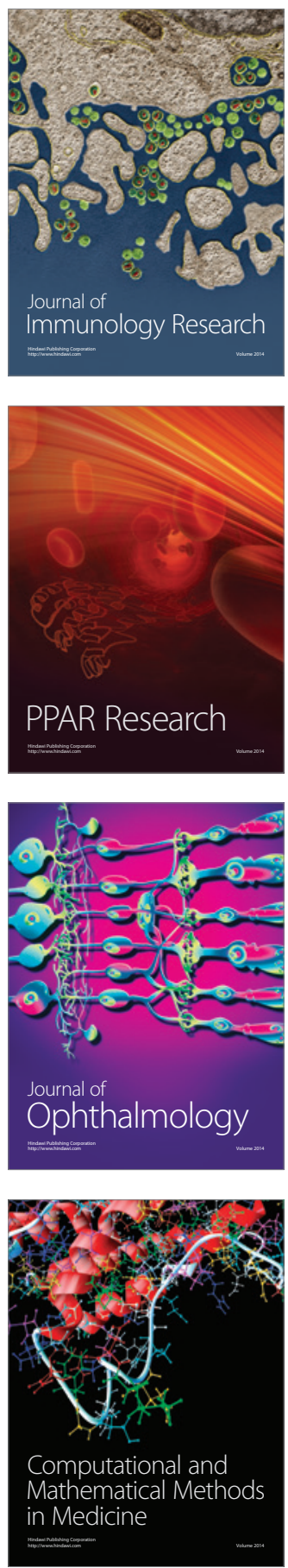

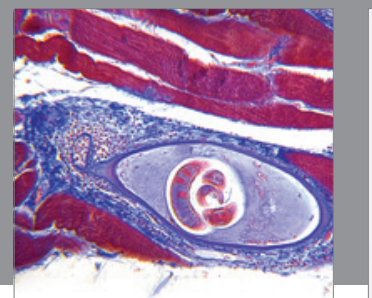

Gastroenterology

Research and Practice
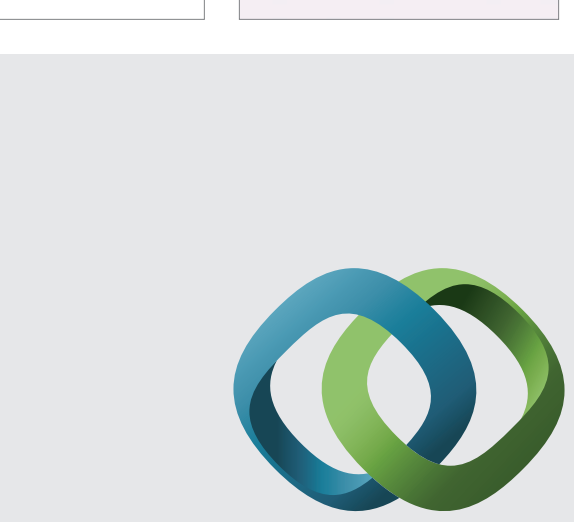

\section{Hindawi}

Submit your manuscripts at

http://www.hindawi.com
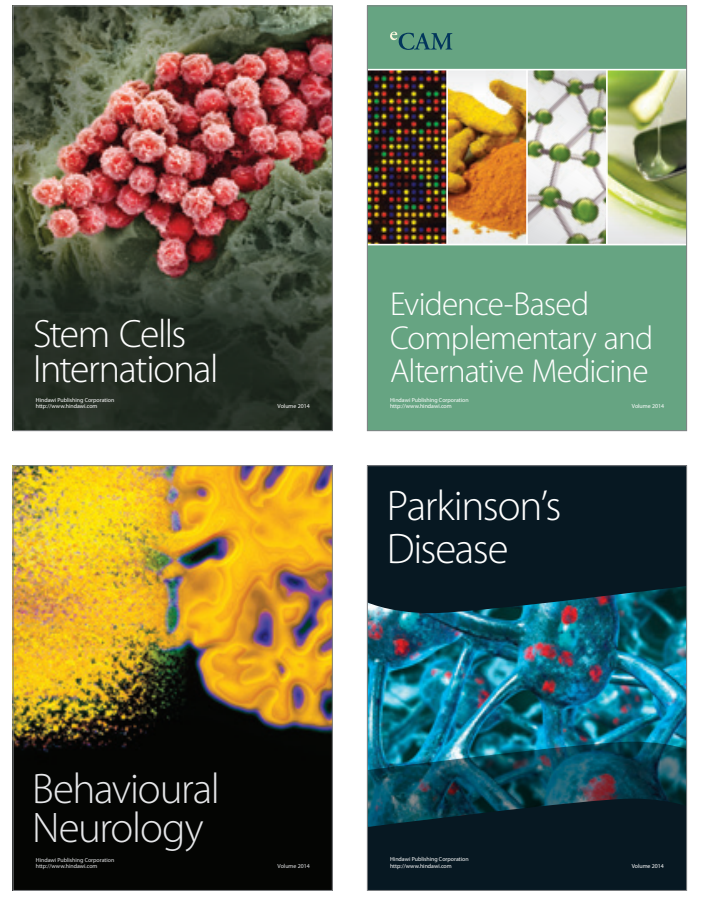
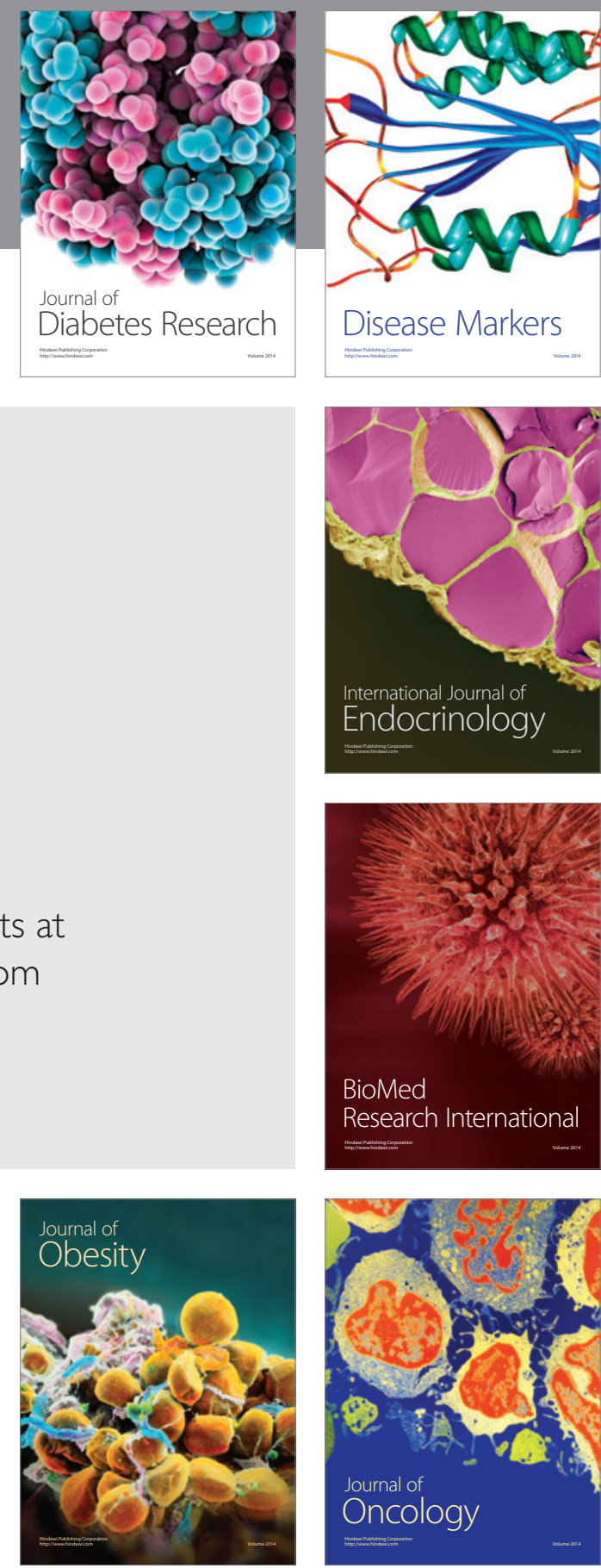

Disease Markers
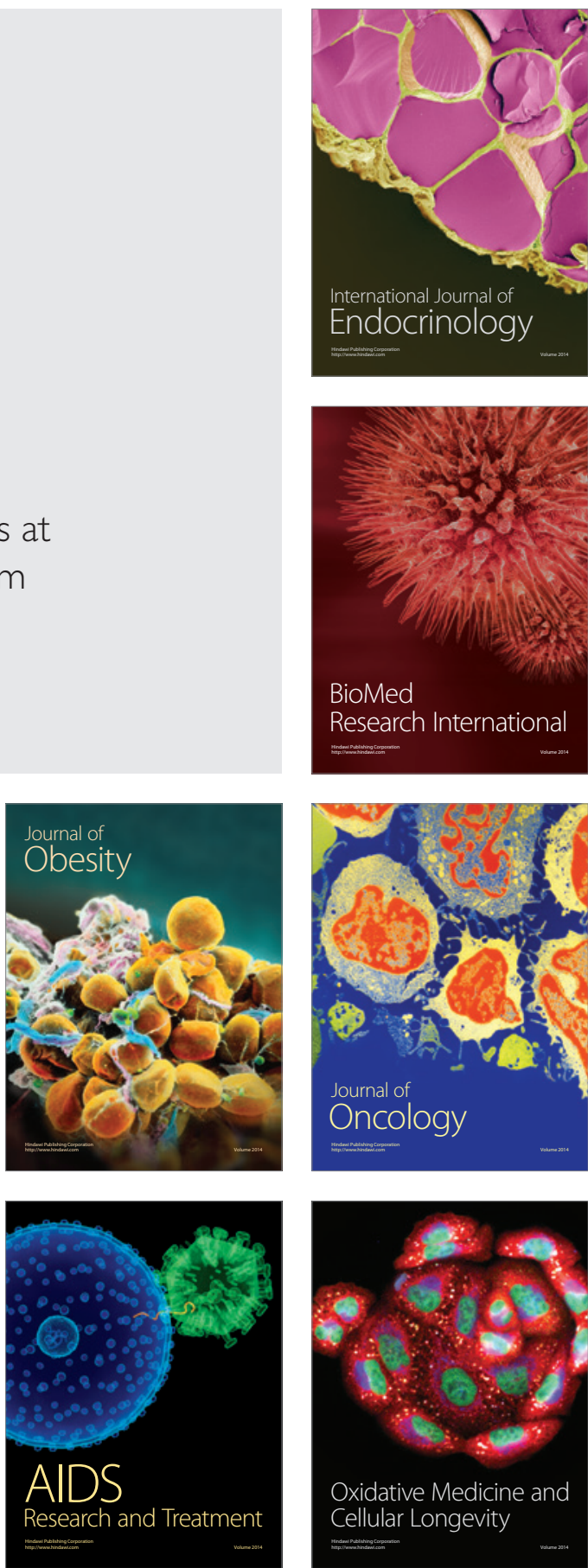